\title{
Cosmology and the Subgroups of Gamma-ray Bursts
}

\author{
A. Mészáros, J. Řípa, L. G. Balázs, Z. Bagoly, P. Veres, I. Horváth
}

\begin{abstract}
Both short and intermediate gamma-ray bursts are distributed anisotropically in the sky (Mészáros, A. et al. ApJ, 539, 98 (2000), Vavrek, R. et al. MNRAS, 391, 1741 (2008)). Hence, in the redshift range, where these bursts take place, the cosmological principle is in doubt. It has already been noted that short bursts should be mainly at redshifts smaller than one (Mészáros, A. et al. Gamma-ray burst: Sixth Huntsville Symp., AIP, Vol. 1 133, 483 (2009); Mészáros, A. et al. Baltic Astron., 18, 293 (2009)). Here we show that intermediate bursts should be at redshifts up to three.
\end{abstract}

\section{Introduction}

In several papers, the authors have shown that there are three subgroups of gamma-ray bursts (GRBs); see Horváth, I. et al, ApJ, 713, 552 (2010) and the references therein. The three subgroups are shown in Figures 1-3 for different instruments (BATSE on the Compton Gamma-Ray Observatory, http://heasarc.gsfc.nasa.gov/docs/cgro/batse/; RHESSI satellite, http://science.nasa.gov/missions/ rhessi/; Swift satellite, http://heasarc.nasa.gov/docs/ swift/swiftsc.html).

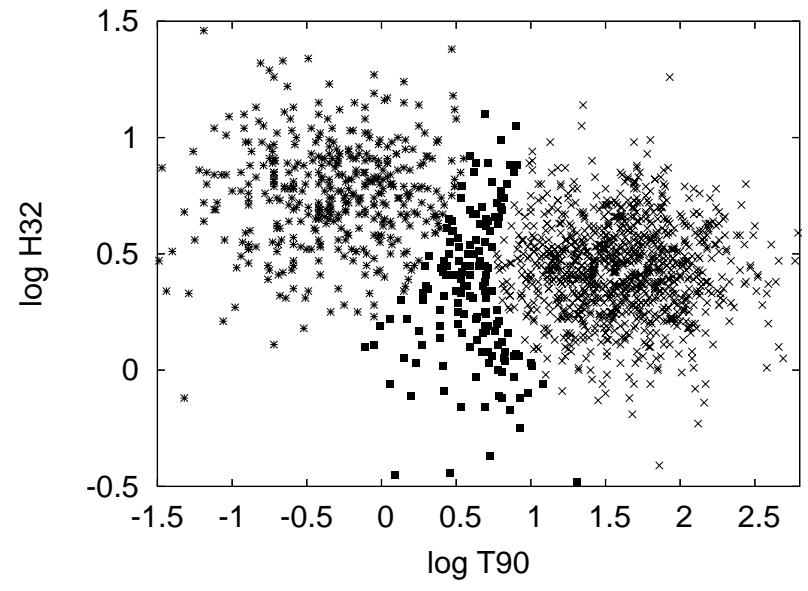

Fig. 1: Three subgroups of BATSE GRBs separated with respect to duration and hardness. T90 is in seconds; for definitions of hardness, duration T90 and for more details see Horváth et al.: $A \& \mathcal{E} A, \mathbf{4 4 7}, 23$ (2006)

\section{Angular distribution of the BATSE GRBs}

The biggest number of detected GRBs is in the BATSE database. If the cosmological principle holds, then they should be distributed isotropically in the sky. In other words, these bursts may well serve as a test of isotropical distribution. These sky distributions are shown in Figures 4-6.

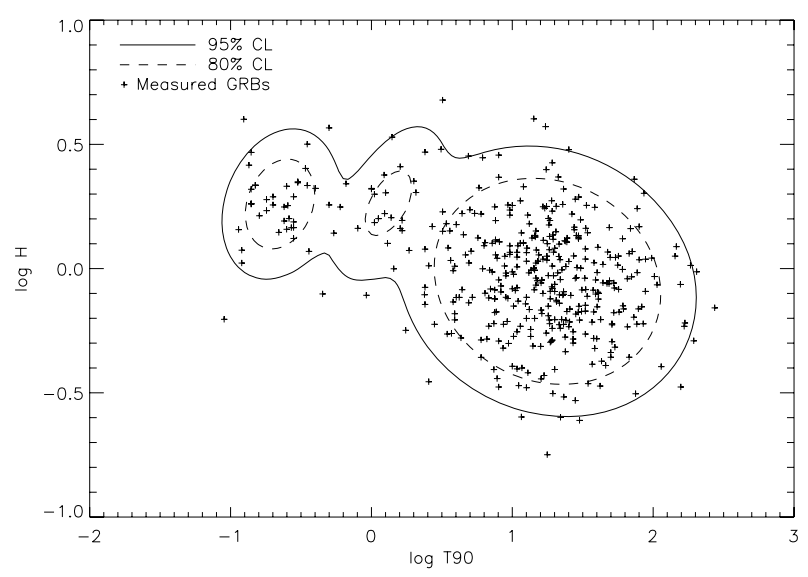

Fig. 2: Three similar subgroups of RHESSI GRBs. T90 is in seconds; Ŕípa et al.: $A \mathscr{G} A$, 498, 399 (2009)

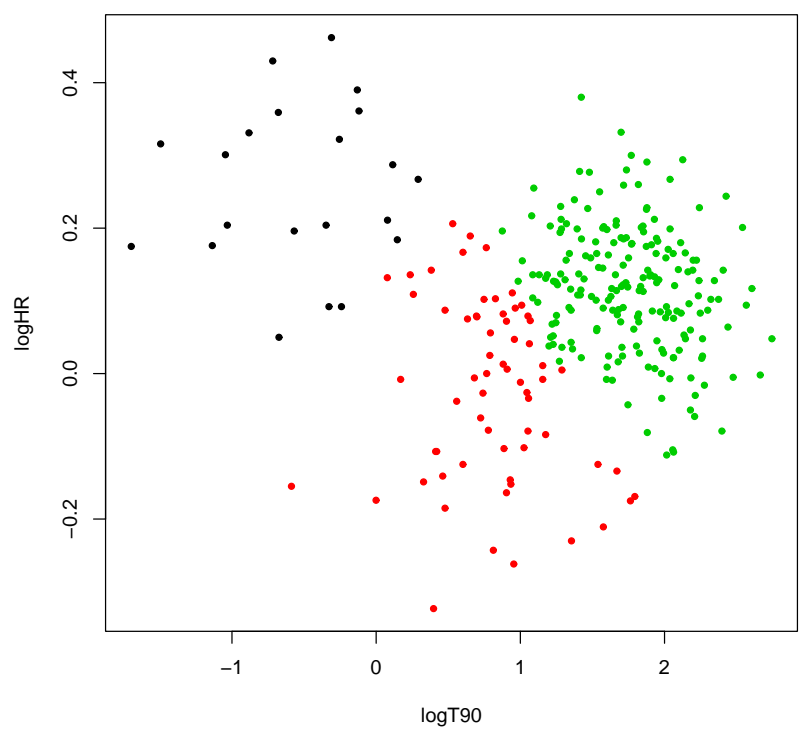

Fig. 3: Three similar subgroups of Swift GRBs. T90 is in seconds; Horváth, I. et al.: ApJ, 713, 552 (2010) 


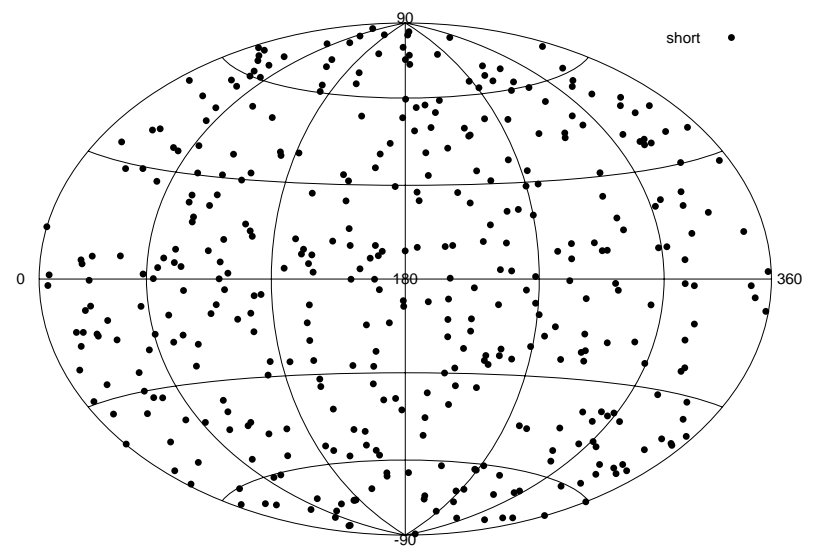

Fig. 4: Celestial distribution of short BATSE GRBs. These short GRBs are not distributed isotropically; Vavrek, R. et al.: MNRAS, 391, 1741 (2008)

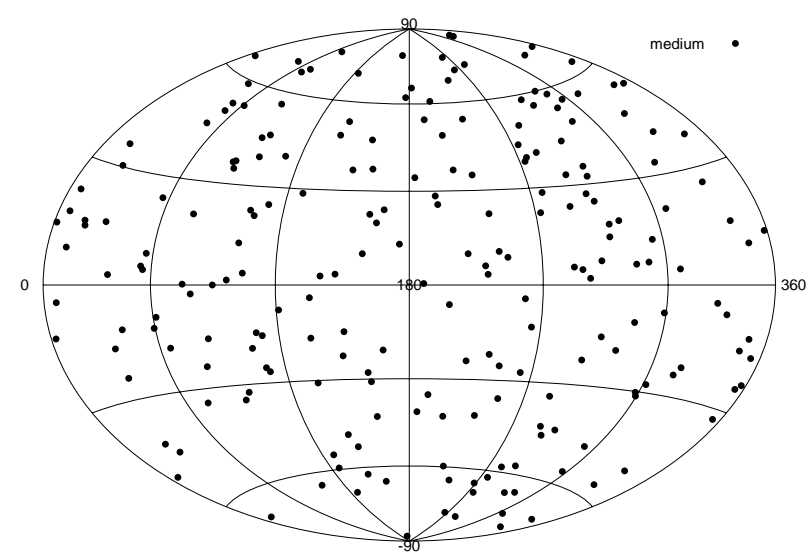

Fig. 5: Celestial distribution of intermediate BATSE GRBs. They are also not distributed isotropically; Mészáros, A. et al.: ApJ, 539, 98 (2000); Vavrek, R. et al.: $M N R A S, \mathbf{3 9 1}, 1741$ (2008)

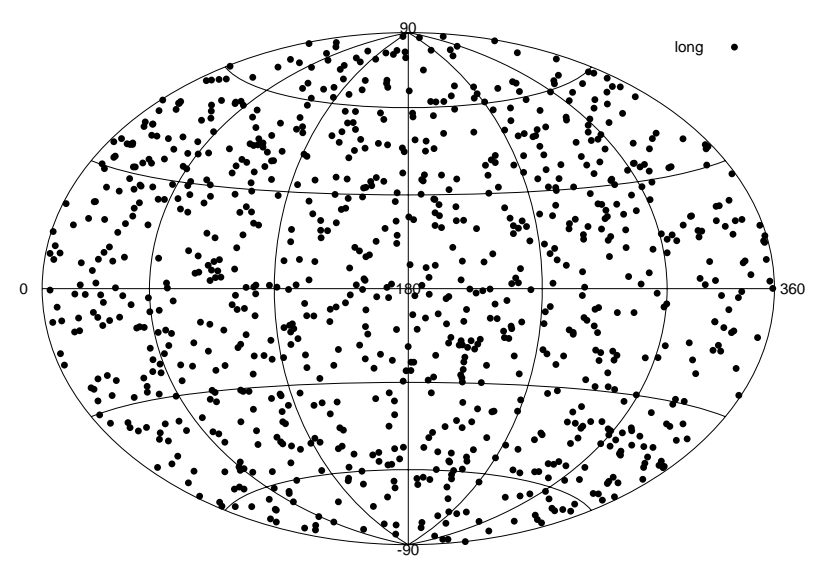

Fig. 6: Celestial distribution of long BATSE GRBs. Long GRBs seem to be distributed isotropically; Vavrek, R. et al.: $M N R A S, \mathbf{3 9 1}, 1741$ (2008)

\section{Redshifts of short and intermediate GRBs}

In two previous papers it was already suggested that short GRBs are mainly at $z<1$ ( $z$ is the redshift); Mészáros, A. et al.: Gamma-ray burst: Sixth Huntsville Symp., AIP, Vol. 1 133, 483 (2009); Mészáros, A. et al.: Baltic Astron., 18, 293 (2009). Hence, up to $z \sim 1$ the cosmological principle is in doubt.

In this paper we have also acquired the redshifts of intermediate GRBs (from the Swift satellite; Horváth, I.: et al, ApJ, 713, 552 (2010)). The redshifts of known Swift GRBs are shown in Figure 7. It is seen that these redshifts are up to $z \sim 3$.

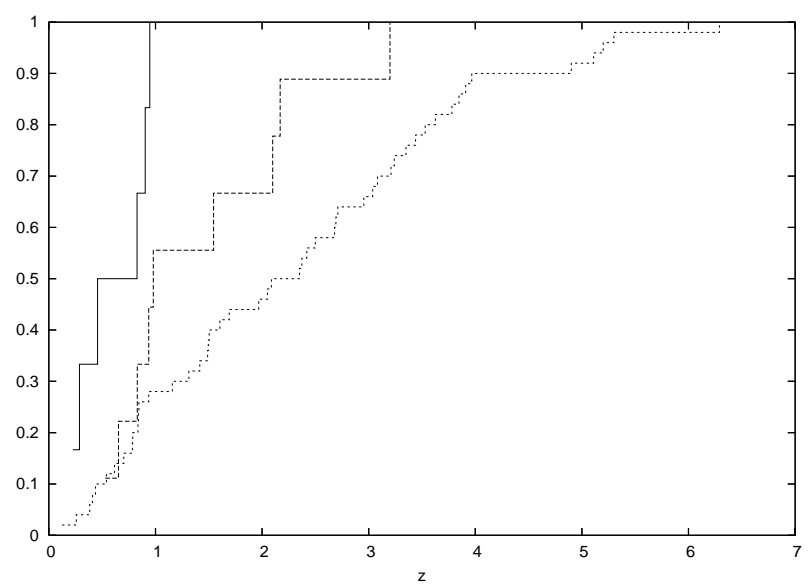

Fig. 7: Redshift distributions of Swift GRBs (short GRBs = solid line; intermediate GRBs = dashed line; long GRBs = dotted line). Intermediate GRBs are at even higher redshifts than short ones; Horváth, I. et al.: ApJ, 713, 552 (2010)

\section{Conclusion}

Of course, it is not fully necessary that the redshifts of intermediate GRBs from two different experiments (BATSE vs. Swift) are the same in the statistical sense. However, keeping this eventuality in mind, it seems that anisotropies exist up to $z \sim 3$ in the spatial distribution of GRBs.

\section{Acknowledgement}

This study was supported by OTKA grant K77795, by Grant Agency of the Czech Republic grants No. 205/08/H005 and P209/10/0734, by project SVV 261301 of Charles University in Prague, and by Research Program MSM0021620860 of the Ministry of Education of the Czech Republic.

\section{References}

[1] Horváth, I., et al.: $A \& A, 447,23$ (2006). 
[2] Horváth, I., et al.: ApJ, 713, 552 (2010).

[3] http://heasarc.gsfc.nasa.gov/docs/cgro/batse/

[4] Mészáros, A., et al.: ApJ, 539, 98 (2000).

[5] Mészáros, A., et al.: Gamma-ray burst: Sixth Huntsville Symp., AIP, Vol. 1 133, 483 (2009).

[6] Mészáros, A., et al.: Baltic Astron., 18, 293 (2009).

[7] Řípa, J., et al.: $A \& \mathcal{G}$ A, 498, 399 (2009).

[8] http://heasarc.nasa.gov/docs/swift/swiftsc.html.

[9] Vavrek, R., et al.: MNRAS, 391, 1741 (2008).

Attila Mészáros

E-mail: meszaros@cesnet.cz

Charles University

Faculty of Mathematics and Physics

Astronomical Institute

V Holešovičkách 2, 18000 Prague 8, Czech Republic

Jakub Rípa

E-mail: ripa@sirrah.troja.mff.cuni.cz

Charles University

Faculty of Mathematics and Physics

Astronomical Institute

V Holešovičkách 2, 18000 Prague 8, Czech Republic
Lajos G. Balázs

E-mail: balazs@konkoly.hu

Konkoly Observatory

PO BOX 67, H-1525 Budapest, Hungary

Zsolt Bagoly

E-mail: zsolt@yela.elte.hu

Lab. for Information Technology

Eötvös University

Pázmány P. s. 1/A, H-1518 Budapest, Hungary

Péter Veres

E-mail: veresp@elte.hu

Lab. for Information Technology

Eötvös University

Pázmány P. s. 1/A, H-1518 Budapest, Hungary

István Horváth

E-mail: Horvath.Istvan@zmne.hu

Dept. of Physics

Bolyai Military University

H-1581 Budapest, POB 15, Hungary 